\title{
Metacognition and abstract reasoning
}

\author{
Henry Markovits • Valerie A. Thompson • Janie Brisson
}

Published online: 22 November 2014

(C) Psychonomic Society, Inc. 2014

\begin{abstract}
The nature of people's meta-representations of deductive reasoning is critical to understanding how people control their own reasoning processes. We conducted two studies to examine whether people have a metacognitive representation of abstract validity and whether familiarity alone acts as a separate metacognitive cue. In Study 1, participants were asked to make a series of (1) abstract conditional inferences, (2) concrete conditional inferences with premises having many potential alternative antecedents and thus specifically conducive to the production of responses consistent with conditional logic, or (3) concrete problems with premises having relatively few potential alternative antecedents. Participants gave confidence ratings after each inference. Results show that confidence ratings were positively correlated with logical performance on abstract problems and concrete problems with many potential alternatives, but not with concrete problems with content less conducive to normative responses. Confidence ratings were higher with few alternatives than for abstract content. Study 2 used a generation of contrary-to-fact alternatives task to improve levels of abstract logical performance. The resulting increase in logical performance was mirrored by increases in mean confidence ratings. Results provide evidence for a metacognitive representation based on logical validity, and show that familiarity acts as a separate metacognitive cue.
\end{abstract}

Keywords Conditional reasoning $\cdot$ Metacognition $\cdot$ Abstract reasoning

H. Markovits $(\bowtie) \cdot J$. Brisson

Department of Psychology, Université du Québec à Montréal,

C.P. 8888, Succ A, Montreal, Quebec H3C 3P8, Canada

e-mail: henrymarkovits@gmail.com

V. A. Thompson

Department of Psychology, University of Saskatchewan, Saskatoon,

Saskatchewan, Canada
An important question in the study of human reasoning is how people evaluate the adequacy of their own inferential processes. To date, much of the debate about people's logical abilities has focused on the kinds of cognitive processes that are used when making inferences, for example mental models (Johnson-Laird \& Byrne, 1991, 2002) versus probabilistic models (Oaksford \& Chater, 2003). In this paper, we shift focus to a different level of analysis, namely the nature of people's metacognitive skills and the processes that they use to evaluate the adequacy of their reasoning performance.

\section{Metacognition and reasoning}

Ackerman and Thompson (2014) have developed a framework for understanding metacognitive processes in the context of reasoning. They used the phrase "meta-reasoning" to refer to the processes that monitor and control reasoning, problem solving and decision-making. Meta-reasoning refers to second-order processes that monitor and control object level cognitive processes (c.f., Nelson \& Narens 1990). In other words, the study of meta-reasoning is not necessarily concerned with inferential processes per se, but with processes that determine how satisfied one is with a conclusion, as well as those that determine how one allocates resources to a problem.

Indeed, it is important to distinguish between debates about the nature of inferential processes and debates about the processes involved in people's metacognitive representations. Specifically, the nature of people's underlying inferential processes determines the kind of information that is used to make specific inferences and potentially whether these inferences will be consistent with the norms of logical validity or not. However, the nature of these processes need not have any direct bearing upon the nature of metacognitive understanding. Indeed, in keeping with other metacognitive theorists, we 
assume that people do not necessarily have access or insight into their cognitive processes when making metacognitive judgments, and that, instead, such judgments are inferential and based on cues (See Dunlosky \& Tauber, 2014; Koriat, 2007 for reviews). Consequently, from a meta-level perspective, the questions are not necessarily about inferential processes per se, but about the cues that are used to evaluate those inferences. From this perspective, one of the more important questions would be what determines whether reasoners are content with a given inference, which involves understanding the processes by which people evaluate their confidence in reasoning outcomes.

Cue-driven confidence in reasoning outcomes Evidence is accumulating to suggest that confidence in reasoning, like confidence in other cognitive outputs, is cue-based and driven by experiences associated with solving the problem, such as fluency. Fluency creates a sense of positive affect (Winkielman \& Cacioppo, 2001), which then forms the basis of a judgment about one's cognitive experiences (see Topolinski, 2014 for a recent review). In the reasoning domain, Thompson and colleagues (Thompson et al., 2013a, Thompson, Evans, \& Campbell, 2013b, Thompson, Prowse Turner, \& Pennycook, 2011) have shown that the fluency with which an initial answer to a reasoning problem is produced is a strong predictor of the "feeling of rightness" that reasoners will have in that answer, which, in turn, predicts the probability that the answer will be reconsidered and changed. Akerman and Zalanov (2012) extended this work to show that answer fluency predicts confidence in problem solutions independently of the correctness of the answer; that is, fluency predicted confidence equally well for correct and incorrect answers. As we outline in the introduction to Experiment 1, one of the goals of this paper was to provide evidence that confidence is sensitive to another potential cue, namely the familiarity of the materials that are being reasoned about.

Meta-representation of validity? In addition, however, we asked whether or not people have a more abstract standard of evaluation. That is, fluency and familiarity are both local cues to confidence, having to do with the quality of the experience associated with reasoning or the properties of the material being reasoned about. However, do people have a more abstract representation of concepts such as logical validity that they can use to evaluate their reasoning performance? And if they do, what information does it represent?

We investigated three hypotheses. The first is that people have a representation of logical validity that extends across contexts. If they do, then their confidence judgments would correlate with logical validity across contexts. Alternatively, it is possible that people's evaluation of conclusions is based on how well the conclusion accords with prior knowledge or belief. A third hypothesis is that the basis of evaluation changes as a function of the content of the material that is being reasoned about. We outline the rationale for each of these hypotheses below.

\section{Validity-based meta-representation}

Developmental studies show that children and adolescents have an increasing ability to explicitly articulate important characteristics of logical reasoning, such as the difference between empirical fact and logical validity (Moshman \& Franks, 1986) and understanding when a conclusion follows from premises (Markovits \& Vachon, 1989). Critically, young children are able to produce logically correct inferences in certain contexts (Markovits, 2000) at a much earlier age than their metacognitive ability to explicitly evaluate these inferences. Developmental studies thus suggest that inferential abilities are independent of metacognitive representations. In fact, Moshman has claimed that the development of reasoning in older reasoners is mostly instantiated by their increased metacognitive abilities (Moshman, 1998). However, while clearly showing the development of the ability to explicitly articulate important aspects of logical validity, there is no evidence that this knowledge is used to monitor people's inferential performance.

One such argument for the existence of a metarepresentation of logic comes from studies conducted in the context of dual process theories. These theories postulate the existence of two inferential systems (Epstein, 1994; Evans, 2007; Sloman, 1996; Stanovich \& West, 2000). The heuristic (Type 1) system makes rapid, low-cost inferences that rely on a variety of heuristics and associative evaluations. The analytic (Type 2) system uses working-memory intensive processes that at least potentially allow the use of abstract logical rules. Dual process theories generally assume that an inferential response involves some degree of competition between these two systems. Since the heuristic system functions automatically and rapidly, a key component of any such competition is the ability to inhibit a heuristic response when the analytic response is different. One problem with this model is that detection of any conflict seemingly requires generation of the analytic response, and some form of comparison between the analytic and heuristic responses. As Morsanyi and Handley (2012) point out, this appears to contradict the whole point of heuristic processing, which must be designed to produce likely conclusions rapidly. One way of resolving this contradiction would be to suppose that people have a form of logical intuition that allows them to detect the presence of a non-logical response, without fully deploying the analytic system.

There is in fact evidence that supports this latter model. Research by De Neys and collaborators (De Neys \& Glumicic, 2008; De Neys, Vartanian, \& Goel, 2008; 
Franssens \& De Neys, 2009) has clearly established in several studies that people who give heuristic responses that are contrary to principles of logic or probability are sensitive to the conflict, without necessarily being able to explicitly express the basis for their judgment. In addition, conflict lowers confidence (De Neys, Cromheeke, \& Osman, 2011; De Neys, Rossi, \& Houdé, 2013; Thompson et al., 2011), suggesting that monitoring judgments are sensitive to this low-level logical analysis. These results are often interpreted as indicating that people possess the ability to make logical inferences quickly; however, they do not provide direct support for the proposition that people are able to form a metacognitive evaluation of the accuracy of their reasoning processes. Similarly, Rips (2001) and Heit \& Rotello (2010) provided evidence that reasoners can distinguish between deductive and inductive arguments, suggesting they are able to discriminate logical validity from other forms of argument strength. However, since these studies did not use any explicit metacognitive evaluations, they do not allow any conclusions about the relationship between inferential performance and metacognitive evaluations.

Studies that have used a direct metacognitive evaluation provide mixed evidence in favor of an abstract metarepresentation of logic. On complex syllogisms, Shynkaruk and Thompson (2006) found weak to zero correlations between confidence and accuracy; indeed, confidence and accuracy appeared to be mediated by different variables. For example, confidence in an answer increased over time, even when people changed responses from a logically valid response to an invalid response. Critically, even amongst those participants who showed a level of logical competence above chance, the relationship between confidence and logical performance remained null. Similarly Prowse Turner and Thompson (2009) found that extensive training with syllogistic problems increased logical accuracy, but had little effect on the ability to discriminate logically correct from incorrect inferences. In other words, these results, which are the most direct evaluations of the relationship between metacognitive representations and inferential performance, clearly suggest that people are often able to make logically normative inferences without being able to metacognitively differentiate these from incorrect inferences.

Other results are less pessimistic. Ackerman and Zalmanov (2012) found that confidence in solutions to misleading nonlogical problems (such as those in Frederick's (2005) Cognitive Reflection Test) was higher for correct than incorrect solutions, even after controlling for fluency. Similarly, on relatively simple logic problems (e.g., conditional inference), Thompson et al. (2011) found a modest, positive relationship between confidence and accuracy. These problems, however, used concrete content, leaving open the possibility that the observed relationship might depend on specific concrete characteristics of the problems that are not necessarily related to logical validity. Thus, the clearest indicator of the ability to make a metacognitive evaluation based on logical norms would require observing a positive relationship between metacognitive evaluations and the validity of specific inferential judgments with abstract content. However, to our knowledge, there is little or no direct evidence of such a relationship, and, in fact, as we have shown above, there is some evidence to the contrary.

\section{Knowledge-based meta-representation}

There is a great deal of evidence that people use their knowledge about familiar premises in order to make inferences, and that this knowledge can determine whether specific inferences correspond to logical norms or not. The clearest example of this concerns conditional ("if then") inferences. Many studies have found that the specific inferences that people make with conditional premises vary systematically according to the empirical properties of these premises, even when the premises are conceptually and pragmatically identical.

One such example is reasoning with causal conditional premises of the form "If cause P then effect Q." These premises can be categorized by the relative number of alternative antecedents (causes of $\mathrm{Q}$ that are different from $\mathrm{P}$ ) that are readily available in semantic memory (Cummins, Lubart, Alksnis, \& Rist, 1991; Markovits \& Vachon, 1990; Thompson, 1994). These studies show that such information affects the kinds of responses made to the two conditional inferences for which there are no logically valid conclusions; the affirmation of the consequent (AC: $\mathrm{P}$ implies $\mathrm{Q}, \mathrm{Q}$ is true) inference and the denial of the antecedent (DA: $\mathrm{P}$ implies $\mathrm{Q}, \mathrm{P}$ is false) inference. Reasoning with conditionals that allow for relatively many potential alternative antecedents tends to produce rejections of the AC and the DA inferences, which is the logical norm. By contrast, reasoning with premises which allow for few potential alternative antecedents tends to produce acceptance of these inferences, which does not correspond to the logical norm. Importantly, these effects remain even when the semantics of the conditional relationship is the same. For example, this has been found for causal conditional relations (Cummins et al., 1991), for deontic conditionals (Thompson, 1994, 2000), and for categorical conditionals (Markovits, 2000). In other words, people's knowledge about potential alternative antecedents influences the way that they make inferences, even within the same semantic class.

Consequently, any correlation between confidence and validity with concrete content may be an artefact of the relative number of alternative antecedents, which is in turn related to people's knowledge of premise content. That is, instead of evaluating confidence on the basis of a meta-representation of logical validity, reasoners may, instead, evaluate the degree to which the conclusion accords with their knowledge of the premises. In that case, confidence would be expected to be 
higher when inferences accord with people's knowledge of premises than when it does not. Consistent with this hypothesis, Thompson et al. (2011; Experiment 1) reported a correlation of .44 ( $p<.01, N=64$ items) between the believability of a conclusion and "feelings of rightness" (FORs) on a conditional reasoning task, where believability was coded to reflect the empirical structure of the premises. However, because FORs were also correlated with validity of the inference and accuracy of response, it is not clear which of the variables in question underlay that correlation. Moreover, one limitation of the knowledge-based hypothesis is that it does not make predictions about how people reason with abstract premises, in which such knowledge is absent.

Rationale for current studies

In summary, we have presented two models for how people might evaluate confidence in logical deductions. The first suggests that people have a meta-representation of the principles of logical validity which can be used to monitor confidence judgments; this view suggests that for both familiar and abstract materials, confidence judgments should be higher for logically correct than incorrect responses. An alternative is that people use their background knowledge to monitor confidence, such that confidence will be higher for inferences that accord with this knowledge. Such a model would predict a positive relation between confidence judgments and logical validity only for content for which knowledge and validity are mutually consistent. Knowledge-based meta-representations can explain evaluations on inferential performance when empirical knowledge is readily available, but do not allow any predictions about the case where knowledge is absent, such as for abstract materials.

There is however a third possibility. People might have access to both forms of meta-representation, which would be differentially activated depending on problem characteristics. There is, in fact, evidence that people tend to use empirical properties of premises in order to generate likelihood estimates of deductive inferences when these are readily available (Markovits, Lortie Forgues, \& Brunet, 2010). This would suggest that people will use knowledge-based cues to confidence when reasoning with premises for which knowledgebased properties of premises are relatively available (such as relative numbers of alternative antecedents), while validitybased cues would be used otherwise. With this view, logical accuracy and confidence would be correlated for abstract premises, since such premises would primarily activate validity-based cues. For concrete premises, which would primarily activate knowledge-based cues, such a correlation would be a by-product of felicitous content; that is, it would only be observed when the empirical structure of the premises produced answers coincident with logical validity, such as conditionals for which many antecedents are available.
Finally, the two studies reported in this paper provide the first empirical investigation of a related meta-reasoning judgment, called the "Judgment of Solvability" (JOS; Thompson, 2009). JOSs are made prior to attempting a solution, and have been proposed to mediate the allocation of time and resources to a problem (Ackerman \& Thompson, 2014). In the current context, we were interested in investigating the accuracy of these judgments, that is, the extent to which JOS accurately predicted reasoning outcomes.

\section{Study 1}

In order to test these hypotheses, we constructed three kinds of inferential problems that presented the four logical forms of conditional reasoning. These are the Modus ponens (MP) inference (If $\mathrm{P}$ then $\mathrm{Q}, \mathrm{P}$ is true), the Modus tollens (MT) inference (If $\mathrm{P}$ then $\mathrm{Q}, \mathrm{Q}$ is false), the Affirmation of the consequent (AC) inference (If $\mathrm{P}$ then $\mathrm{Q}, \mathrm{Q}$ is true), and the Denial of the antecedent (DA) inference (if $\mathrm{P}$ then $\mathrm{Q}, \mathrm{P}$ is false).

All three problems used conditional relations as the major premise. The first used abstract conditional premises, with nonsensical terms, such as: "If someone glebs, then they are brandup." We also constructed two sets of familiar causal conditional premises, with variable properties determined by the number of potential alternative antecedents. Both sets of premises used relations with the antecedent being a plausible cause for the consequent. This, in combination with standard logical instructions to accept the major premise is true, should lead to relatively high rates of acceptance of the MP and the MT inferences for both forms of familiar premises. The key difference was the relative number of alternative antecedents available. In the Many alternatives condition, we used causal conditionals with familiar content with the antecedent being a plausible but unlikely cause for the consequent, e.g. "If a candle is lit, the room will be illuminated." These conditionals were designed so that it is possible to produce alternative antecedents that are more likely causes. For example, turning on a light or opening a window are common ways to illuminate a room. The ready availability of these counter-examples should suggest rejection of the AC and DA inferences (as per Cummins et al., 1991; Markovits \& Vachon, 1990; Thompson, 1994). In the Few alternatives condition, premises used concrete causal conditionals with the antecedent being a very strong cause for the consequent, with few common alternative antecedents easily available, e.g. "If someone cuts their finger, the finger will bleed." Such premises lead to relatively high rates of acceptance of the AC and DA inferences.

To summarize, all three premise types presented causal conditional relations. The key difference was the availability 
of empirical knowledge of premises and its nature. The abstract premises used nonsensical terms for which people would have no empirical knowledge base. Both of the familiar conditionals did present such a knowledge base. However, in this case, premises were designed so that simple use of empirical knowledge should generate relatively higher rates of responses that correspond to a standard conditional interpretation on the "Many alternatives premises" than on the "Few alternatives premises."

Each of the three meta-representation models described previously suggest different patterns of relations between people's metacognitive evaluations and logically correct responding for the three different problem types (logically correct responses involve accepting the MP and the MT inferences, and rejecting the $\mathrm{AC}$ and the $\mathrm{DA}$ inferences). A knowledge-based meta-representation would lead to different patterns of correlations for the Many and Few alternatives conditions. Because the premises used in the Many alternatives condition were designed to generally produce logically correct responses (with a high probability of the MP and the MT inferences, and a low probability of the AC and the DA inferences), this would suggest that there should be a positive relationship between metacognitive evaluations and the rate of logically correct responses for these premises. By contrast, since the premises used in the Few alternatives condition were designed to produce lower levels of logically correct responses (particularly with higher probabilities of accepting the $\mathrm{AC}$ and the DA inferences), the relationship between metacognitive evaluations and logical norms should be less strong for these premises. Finally, since premises with abstract content do not provide any knowledge-based indications of conclusion likelihood, this hypothesis would not predict any positive relation between metacognitive evaluations and logical norms.

If, instead, reasoners rely on a validity-based representation to evaluate confidence, then we would expect that metacognitive evaluations should reflect the extent to which inferences correspond to logical norms, irrespective of premise content. Finally, a dual meta-representation model supposes that reasoning with abstract content will result in preferential use of a validity-based meta-representation, while reasoning with familiar content will result in preferential use of a knowledge-based representation. This model predicts that there should be a positive relation between metacognitive evaluations and rates of logically correct responses for abstract premises and that when reasoning with familiar premises, the relationship between metacognitive evaluation and logical norms will vary according to the knowledge-based properties of the premises. Thus, there should be a positive relationship between metacognitive evaluations and rates of logically correct responses for the Many alternatives premises, but not for the Few alternatives premises.
In this study, we examined two kinds of metacognitive judgments about reasoning with both abstract and concrete premises. Participants were first given a brief description of what is involved in making logical inferences with no specification of premise content. They were then asked for an evaluation of their level of confidence that they would be able to give "logical" responses. This judgment was done in the absence of any contextual cues, and corresponds to what Thompson (2009) has called Judgments of Solvability (JOSs), as mentioned above. These initial JOS ratings should reflect people's basic beliefs about the criteria involved in making logical inferences irrespective of content. Since these beliefs are accessed before any specific forms of reasoning, a differential pattern of relationship between JOS ratings and accuracy would be consistent with our general hypothesis that metacognitive strategies are modified by problem content.

Subsequently, participants were given a sequence of reasoning problems each of which was followed immediately by a measure of their Confidence in the response, which was modelled after the FOR measure used by Thompson et al. (2011). Confidence ratings are contextualized and reflect the reasoner's immediate evaluation of the efficacy of the actual inferential processes used in this context.

Differences in the nature of the premises allowed us to test another hypothesis about the basis of confidence in reasoning. As we outlined in the introduction, there is good evidence that metacognitive judgments are informed by local cues, such as the fluency with which an answer is generated (Ackerman \& Zalmanov, 2012; Thompson, et al., 2011; Topolinski, 2014). The current study allowed us to test the hypothesis that familiarity of the material being reasoned about is another such cue, such that familiarity would increase confidence in responses, as it does in other domains, such as metamemory (Costermans, Lories, \& Ansay, 1992; Koriat \& Levy-Sadot, 2001). Consistent with this hypothesis, previous results have suggested that confidence ratings are higher when reasoning with familiar content (Shynkaruk \& Thompson, 2006). However, these results confounded familiarity with inferential performance, in that accuracy also differed between the familiar and unfamiliar materials. The design of our study allowed us to rule out difference in accuracy as a basis for any potential effect of familiarity, as we examined performance with familiar materials designed to both enhance (many alternatives) and reduce (few alternatives) logical performance.

\section{Method}

Participants A total of 235 university students (98 men, 137 women: average age $=21$ years, 5 months) participated in this study. All participants were volunteers, and were students at the Université du Québec à Montréal. 
Procedure Questionnaires were randomly distributed to participants within entire classes. Participants were told to read the instructions carefully and to take as much time as required.

Materials Three five-page booklets were prepared corresponding to the three experimental conditions. On the first page of each, participants were asked to give their gender and age. Directly following (and on the same page), all versions presented the following instructions (all instructions are translated from the original French):

"In the following pages, you will be presented with several rules. You must consider these to be true. After each rule, an observation and a conclusion will be presented. You will be asked to indicate if the conclusion follows logically or not from the information given." Following this, participants were asked to rate their initial level of confidence in their ability to make logical responses (JOS):

"What is your degree of confidence that your responses will be generally logical?"

Directly below this question, participants were presented with a 7-point scale, ranging from $1=$ not at all confident to 7 $=$ absolutely confident.

In the abstract condition, on the next pages, participants received eight abstract reasoning problems. The first four used the major premise: "If someone glebs, then they are brandup." The second four used the major premise: "If something mobs, then it blorqs." For each problem, the major premise was presented with the instruction to consider that it is true. Following this, participants were presented with one of four minor premises, corresponding to the logical forms MP ("A person glebs"), AC ("A person is brandup"), DA ("Someone does not gleb"), MT ("a person is not brandup"), in that order. We chose this specific order to reflect the relative complexity of the logical forms, going from simplest with only positive terms to the more complex forms involving negations. This was done in order to minimize any interactions between problem complexity and confidence ratings. For each of the two major premises, the MP and AC inferences were presented on a single page, while the DA and MT inferences were presented on the next page.

For each combination of major and minor premises, participants were given three response choices, corresponding to a positive conclusion, a negative conclusion, and uncertainty. As an example of the specific presentation, the following is how the AC inference was presented following the major premise, "If someone glebs, then they are brandup":

A person is brandup. One can conclude that:

1) The person glebs.

2) The person does not gleb.

3) One cannot conclude if the person glebs or not.
Immediately after each response, participants were presented with a 7-point FOR scale asking for their immediate feeling when giving this response, ranging from $1=$ guessing to $7=$ certain to be correct. This was presented as follows:

"At the moment that I gave my answer, I felt:

1 = guessing; 2, 3, 4 = somewhat certain, 5, 6, 7 = certain

that I was right"

Note that the points corresponding to 2,3,5, and 6 were not given an explicit description in the scale.

The Many alternatives condition used the following two major premises:

"If a person meditates, then they will feel rested"

"If a candle is lit, then the room will be illuminated"

The Few alternatives condition used the following two major premises:

"If a finger is cut, then the finger will bleed"

"If a dog has fleas, then the dog will scratch constantly"

Order of the minor premises and presentation of the inferences was exactly the same as for the abstract problems. All participants responded to eight inferences, with two of each of the four logical forms. We judged that increasing the number of inferences, while statistically useful, would increase the probability of people changing their criteria for confidence given the high degree of similarity of the problems presented.

In summary, premise content (abstract, many alternatives, few alternatives) is a between-subjects variable while JOS rating, logical performance and confidence ratings are within-subjects variables.

\section{Results}

We first examined performance on the reasoning problems. Each of the eight inferences was coded as being logically correct or not (1 or 0$)$. It should be noted that the logical response was: for the MP inference, "Q is true," for the MT inference, "P is false," while uncertainty was the logical response for the $\mathrm{AC}$ and DA inferences. It should be noted that although there were three response options, almost all participants used only two of these; responding with either uncertainty or the biconditional inference (MP: "Q is true", MT: "P is false", AC: "P is true", $\mathrm{DA}$ : " $\mathrm{Q}$ is false"). We calculated the number of logically correct responses (out of a total of two) and the main confidence score for each of the four logical forms (see Table 1). We also constructed a total Logical score by summing the number of logically correct responses across all eight problems.

We first examined logical reasoning as a function of problem content in order to verify that the effects of our content 
Table 1 Mean number of logically correct responses (with a maximum of two) and mean confidence ratings (varying between 1 and 7 ) for the four conditional inferences (MP, MT, AC, DA) by content

\begin{tabular}{|c|c|c|c|c|c|}
\hline Premise content & & MP & $\mathrm{AC}$ & DA & MT \\
\hline \multirow[t]{2}{*}{ Abstract } & Correct & $1.62(0.69)$ & $1.12(0.90)$ & $0.92(0.93)$ & $0.99(0.90)$ \\
\hline & Confidence & $5.03(1.85)$ & $4.88(1.86)$ & $4.71(1.78)$ & 4.69 (1.77) \\
\hline \multirow[t]{2}{*}{ Many alternatives } & Correct & $1.62(0.63)$ & $1.65(0.70)$ & $1.51(0.72)$ & $1.05(0.83)$ \\
\hline & Confidence & $6.12(0.82)$ & $6.19(0.94)$ & $6.02(1.05)$ & $6.05(1.02)$ \\
\hline \multirow[t]{2}{*}{ Few alternatives } & Correct & $1.56(0.59)$ & $1.40(0.74)$ & $0.99(0.86)$ & $1.09(0.81)$ \\
\hline & Confidence & $6.23(0.98)$ & $6.09(0.98)$ & $5.99(1.12)$ & $6.07(1.13)$ \\
\hline
\end{tabular}

$M P$ Modus ponens inference (If $\mathrm{P}$ then $\mathrm{Q}, \mathrm{P}$ is true), $A C$ Affirmation of the consequent inference (If $\mathrm{P}$ then $\mathrm{Q}, \mathrm{Q}$ is true), $D A$ Denial of the antecedent inference (if P then Q, P is false), MT Modus tollens inference (If P then Q, Q is false)

manipulation were as expected. We performed an $4 \times 3$ mixed ANOVA with number of logical responses as dependent variable with Logical form (MP, MT, AC, DA) as a repeated measure and Content (Abstract, Many alternatives, Few alternatives) as a between-subjects variable. This gave a significant main effect of Content, $\mathrm{F}(2,232)=12.30, \mathrm{p}<.001$, partial $e t a^{2}=.096$, and Logical form, $\mathrm{F}(3,232)=23.95, \mathrm{p}<.001$, partial eta ${ }^{2}=.094$, and a significant interaction between Content $\times$ Logical form, $\mathrm{F}(6,232)=3.58, \mathrm{p}<.001$, partial $e t a^{2}=.030$. Follow-up analyses used one-way ANOVAs for each of the four logical forms. For the MP and the MT forms, no significant effect of content was observed. For the AC form, there was a significant effect of Content, $F(2,232)=$ $8.87, \mathrm{p}<.001$, partial eta ${ }^{2}=.071$. Post hoc comparisons used the Student Newmann-Keuls procedure with $\mathrm{p}=.05$. This showed that the number of logically correct responses was less for the Abstract premises $(\mathrm{M}=1.12, \mathrm{SD}=0.90)$ than for the Few alternatives premises $(\mathrm{M}=1.40, \mathrm{SD}=0.74)$, which in turn was less than the number for the Many alternatives premises $(\mathrm{M}=1.65, \mathrm{SD}=0.70)$. For the $\mathrm{DA}$ form, there was a significant effect of Content, $F(2,232)=11.25, p<$ .001 , partial eta ${ }^{2}=.088$. Post hoc comparisons showed that the number of logically correct responses was greater for the Many alternatives premises $(\mathrm{M}=1.51, \mathrm{SD}=0.72)$ than for the Few alternatives premises $(\mathrm{M}=.99, \mathrm{SD}=0.86)$ and for the Abstract premises $(\mathrm{M}=.92, \mathrm{SD}=0.93)$. In other words, the observed differences accurately correspond to predicted differences based on previous studies examining the effects of content on inferential reasoning (e.g. Cummins et al., 1991; Thompson, 2000).

We then examined confidence ratings. We performed a $4 \times$ 3 mixed ANOVA with mean Confidence rating as dependent variable and Logical form as repeated measure and Content as independent variable. This showed significant main effects of Logical form, $\mathrm{F}(3,232)=4.91, p<.01$, partial eta ${ }^{2}=.060$, and of Content, $\mathrm{F}(2,232)=28.77, \mathrm{p}<.001$, partial eta ${ }^{2}=$ .119. Post hoc analysis was done using the Student NewmannKeuls procedure with $p$ equals .05 . This showed that mean confidence ratings were higher for the $\mathrm{MP}(\mathrm{M}=5.80, \mathrm{SD}=$
1.40) and the $\mathrm{AC}(\mathrm{M}=5.73, \mathrm{SD}=1.44)$ inferences than for the DA $(\mathrm{M}=5.59, \mathrm{SD}=1.48)$ and the $\mathrm{MT}(\mathrm{M}=5.61, \mathrm{SD}=$ $1.49)$ inferences. Mean confidence ratings were higher on the Many alternatives $(\mathrm{M}=6.10, \mathrm{SD}=0.85)$ and the Few alternatives $(\mathrm{M}=6.09, \mathrm{SD}=0.89)$ than on the abstract premises $(\mathrm{M}=4.80, \mathrm{SD}=1.74)$. Although confidence ratings generally showed consistently high levels of confidence (around 5), these results confirm our prediction that reasoning with familiar content produces significantly higher confidence levels, even when it lowered accuracy of performance.

Examination of the pattern of correlations amongst the confidence ratings for specific logical forms showed that these were uniformly very high, ranging between .76 and .88. Moreover, as reported above, the only source of variation in confidence ratings was the difference between inferences involving positive and those involving negative minor premises. We thus considered the overall mean confidence rating as a reasonable measure of participants' immediate evaluations about how reliable their reasoning was. Correspondingly, we calculated an overall Confidence score, as well as a Logic score, which was the mean number of logically correct responses across all eight problems.

We then examined the correlation between Logic scores, mean Confidence rating, and initial JOS rating, for each of the three problem types (see Table 2). Inspection of the table shows that the pattern of correlations between confidence ratings and logical performance is similar to that predicted by the dual meta-representation model. There were positive correlations for both the Abstract and the Many alternatives premises, but not for the Few alternatives premises. This picture is somewhat complicated by the fact that the initial JOS rating was related to confidence levels for all conditions. Consequently, we calculated partial correlations between confidence and logic scores, after accounting for JOSs. This showed two key results. First, and consistent with our hypothesized validity-based meta-representation, there was a significant partial correlation between logical responses and confidence level for the Abstract problems, $r(77)=.221, p=.05$. Second, the partial correlation between confidence and logic 
Table 2 Relations between Judgment of Solvability (JOS), mean Confidence ratings, and Logical reasoning scores as a function of problem content Problem content

\begin{tabular}{|c|c|c|c|c|c|c|}
\hline & \multicolumn{2}{|l|}{ Abstract } & \multicolumn{2}{|c|}{ Many alternatives } & \multicolumn{2}{|c|}{ Few alternatives } \\
\hline & Confidence & Logic & Confidence & Logic & Confidence & Logic \\
\hline JOS & $.37 * * *$ & $.29 * * *$ & $.31 * * *$ & -.02 & $.35 * * *$ & .16 \\
\hline Confidence & & $.30 * * *$ & & $.32 * * *$ & & .06 \\
\hline
\end{tabular}

was greater for the Many alternatives premises, $\mathrm{r}(77)=.342$, than for the Few alternatives premises, $\mathrm{r}(81)=.002, \mathrm{z}=2.18$, $\mathrm{p}<.05$, as predicted by the knowledge-based meta-representation hypothesis.

Finally, as can be seen by inspection of Table 2, JOS ratings were significantly related to Logic scores only for the Abstract problems. This correlation was higher for Abstract problems than for the Many alternatives problems, $\mathrm{z}=1.97, \mathrm{p}<.05$, while it was non-significant but intermediate in value for the Few alternatives problems. We interpreted this finding to suggest that reasoners' initial evaluation of their ability to make logical inferences draws on a validity-based meta-representation.

One potential problem with these analyses is that they simply use the total of logical responses. This could be misleading, because logical responses could be generated on some inferences in a way that corresponds to a non-standard way of processing the premises. For example, people who interpret conditionals as bi-conditionals will give the logically correct response to the MP and MT inferences. In order to examine in more detail what underlies these patterns, we constructed measures that captured three different patterns of responses. The Conditional score was constructed to reflect the extent to which inferences conformed to a standard conditional interpretation. For the conditional pattern, we determined if the MP inference was accepted. If so, we added up the total number of logically correct responses on the AC, DA, and MT inferences. This was repeated for each of the two major premises (giving a score between 0 and 6). The Biconditional score was similar, except that if the MP inference was accepted, we added up the total number of the other three inferences that were accepted (giving a score between 0 and 6). We then examined one other pattern that has been observed previously, and that accounts for a reasonably large proportion of responses here. This is an Inverted pattern, where the two valid inferences are refused and the invalid ones are accepted. We thus constructed an Inversion score. For each problem, we determined if the MP inference was refused. We then added up the number of times that the AC or DA inferences were accepted and that the MT inference was rejected (giving a score between 0 and 6). Table 3 indicates mean Conditional, Biconditional, and Inverted scores for the three problem contents.
Inspection of Table 3 shows that the difference in Conditional scores does indeed mirror the effect found by simply adding the total of Logical responses. There was a significant effect of content on these responses, $\mathrm{F}(2,232)=$ $5.69, \mathrm{p}<.01$, partial eta ${ }^{2}=.051$. As expected, Conditional scores were higher with Many alternatives than with the other two contents. No significant differences were observed with the Biconditional and Inverted scores.

We then examined the relation between mean Confidence and these three response patterns for each of the three content types. In line with our previous results, there was a significant, positive correlation between mean Confidence and Conditional scores for both the Abstract, $\mathrm{r}(77)=.35, \mathrm{p}<$ .01 , and the Many alternatives premises, $\mathrm{r}(77)=.28, \mathrm{p}<.02$. By contrast, for the Few alternatives problems, the correlation with Conditional scores was not significant; only the correlation between mean Confidence and Inverted responses was significant, $\mathrm{r}(81)=.26, \mathrm{p}<.02$. These data provide further evidence in favor of the knowledge-based meta-representation, because the relationship between confidence and response patterns varied according to the relative numbers of alternative antecedents associated with the two premise types.

\section{Discussion}

These results provide strong initial support for our predictions. Our first prediction was that confidence ratings would be higher for familiar premises, irrespective of levels of logical performance. This was, in fact, confirmed. Confidence ratings were higher for both the Many alternatives and the Few alternatives premises than for the Abstract premises, with no

Table 3 Mean number of Conditional, Biconditional and Inverted responses as a function of problem content

\begin{tabular}{llll}
\hline & \multicolumn{2}{l}{ Problem content } \\
\cline { 2 - 4 } & Abstract & Many alternatives & Few alternatives \\
\hline Conditional & 2.49 & 3.40 & 2.74 \\
Biconditional & 2.52 & 1.79 & 2.37 \\
Inverted & 0.70 & 1.03 & 1.00 \\
\hline
\end{tabular}


difference between the former. These results are the first to show that confidence levels with familiar premises are generally higher than those with abstract premises, and that this difference persists despite differences in overall levels of logical performance. Strikingly, confidence ratings were higher for both the familiar premises than for the abstract premises even on the two logical forms for which there was no difference in performance related to content (MP, MT). There are several possible mechanisms that might mediate this effect. For example, it is possible that familiarity facilitates fluent processing, which, in turn, produces a feeling of rightness in the answer (Ackerman \& Zalmanov, 2012; Thompson et al., 2011; Topolinski, 2014). Alternatively, familiarity might be an independent cue to confidence, such that the ability to make decisions on the basis of one's prior knowledge, in and of itself, promotes confidence (Shynkaruk \& Thompson, 2006).

Our second prediction concerned the relationship between confidence and inferential performance. Our results are the first to show positive correlations between confidence levels and logical performance for Abstract problems, indicating that people can indeed evaluate the extent to which their inferences are logically valid with Abstract premises. They also show that with familiar premises, this relationship does not always hold. When problems have a knowledge-based pattern that is conducive to production of responses corresponding to logical norms, as is the case with the Many alternatives problems, there was a positive correlation between confidence and logical norms. By contrast, with the Few alternatives problems which were chosen to have a different pattern, this was not the case. These two sets of results are thus consistent with a dual meta-representational model, which supposes that people have both a metarepresentation of logical validity, preferentially used to evaluate abstract content, and one based on knowledge-based cues, which is preferentially activated with familiar content.

Additional evidence for this conclusion comes from the relationship between initial JOSs and accuracy. Remember that JOS ratings access people's metacognitive beliefs about their ability to make logical inferences that is unrelated to specific content. If their basic meta-representational processes remained constant across different problems, we would expect the relationship between JOS ratings and performance also to remain constant. However, this was not the case. The relation between JOS ratings and performance was positive for the Abstract problems but was close to 0 for the familiar problems with Many alternatives, despite the fact that the latter generated much higher levels of responses corresponding to logical norms.

\section{Study 2}

The results of Study 1 are the first to show that people's mean immediate level of confidence in their own abstract reasoning is related to the extent to which this reasoning corresponds to logical norms. However, this study used a correlational design, and in the following, we attempt to provide an additional, experimental test of this idea. Specifically, if people's immediate level of confidence does indeed reflect their ability to reason according to logical norms, we would predict that any manipulation that improves logical reasoning with abstract content should generate an increase in overall confidence ratings. Ideally, such a manipulation should not depend on transmitting specific notions of abstract logic. Fortunately, a recent study suggests just such a method. Markovits and Lortie Forgues (2011) showed that asking older adolescents to generate potential alternative antecedents to empirically false conditionals produced a global improvement in abstract reasoning. This manipulation was designed to promote use of a counterexample generation strategy resulting from a developmental model based on Karmiloff-Smith's (1995) representational redescription theory (see Markovits \& Lortie Forgues, 2011, for details). This manipulation did not specifically require participants to understand logical principles, such as logical necessity, since participants were only asked to generate alternative antecedents, and were given no indication of the relationship between these and validity. It should be noted that this manipulation is similar in some respects to findings that priming an additive counterfactual thinking mindset can improve analytic reasoning on syllogisms with concrete content (e.g., Markman, Lindberg, Kray, \& Galinsky, 2007). Similarly, Galinsky and Moskowitz (2000) observed that priming a counterfactual mindset improved performance on the Wason selection task. The underlying mechanism of these and the present manipulation is related to the idea that logical reasoning can be improved by increasing spontaneous retrieval of potential alternatives.

In order to examine this prediction, we first presented reasoners with the same abstract reasoning problems as were used in the initial study, preceded by an alternative antecedent generation task based on empirically false conditional rules. In order to provide a comparison, we used a control task which was designed to have no impact on reasoning, i.e., naming four African countries. On the same page, and directly following this initial problem, a JOS rating was presented (as in Study 1). Subsequently, participants were given a sequence of abstract reasoning problems with a Confidence rating for each (identical to those used in Study 1).

Method

Participants A total of 119 university students (49 men, 70 women: average age $=24$ years, 7 months) participated in this study. All participants were volunteers, and were students at the Université du Québec à Montréal. 
Procedure Questionnaires were randomly distributed to entire classes. Participants were told to read the instructions carefully and to take as much time as required.

Materials Two six-page booklets were prepared corresponding to the two experimental conditions. On the first page of each, participants were asked for their gender and age. On the top of the subsequent page, one of two problems requiring information generation was presented. In the Contrary to fact (CF) alternatives condition, this problem was the following (translated from the original French):

"Imagine that you are on a planet where everything works exactly opposite to what happens here on earth. On this planet, it is true that:

If a great deal of mustard is put on a shirt, the shirt will become clean.

Below, please indicate 4 things that someone on this planet could do in order to clean a shirt."

In the Control condition, the following problem was given:

"There are many countries in Africa.

Below, please name 4 countries in Africa."

Following this, four numbered lines were presented.

Directly following the lines (and on the same page), both versions presented the following instructions:

"In the following pages, you will be presented with several rules. You must consider these to be true. After each rule, an observation and a conclusion will be presented. You will be asked to indicate if the conclusion follows logically or not from the information given."

On the next pages, participants received the same eight abstract reasoning problems used in the Abstract condition in Study 1 (these were identical in both versions). The presentation of the inferential problems and the Confidence scales were also identical.

In summary, condition ( $\mathrm{CF}$ alternatives, control) is a between-subjects variable and JOS rating, reasoning performance and confidence ratings are within-subjects variables.

\section{Results}

We first examined performance on the abstract reasoning problems. We added up the total number of logically correct responses for each of the four logical forms on the abstract reasoning problems (see Table 3 ). Then we performed a $4 \times 2$ mixed ANOVA with Number of correct responses as dependent variable with Logical form as repeated measure and Condition as a between-subjects variable. This gave significant effects of Condition, $F(1,117)=4.51, p<.05$, partial eta ${ }^{2}=.037$, and logical form, $F(3,117)=16.32, p<$ .001 , partial eta $^{2}=.122$. The interaction between condition $\times$ logical form was nonsignificant, $F<1$. The total number of correct responses was higher in the $\mathrm{CF}$ alternatives condition $(\mathrm{M}=5.33, \mathrm{SD}=1.58)$ than in the control condition $(\mathrm{M}=4.71$, $\mathrm{SD}=1.61)$.

We then examined participants' JOS and mean Confidence ratings as a function of Condition. We performed a $2 \times 2$ mixed ANOVA with Rating type (JOS vs confidence) as repeated measure and Condition as a between-subjects variable. This gave a significant main effect of Rating type, $F(1$, 116) $=14.19, p<.01$, partial eta ${ }^{2}=.042$, and a significant interaction involving Rating type $\mathrm{X}$ Condition, $F(1,116)=$ $3.99, p<.05$, partial eta ${ }^{2}=.117$. Post hoc analyses using Neumann-Keuls tests with $\mathrm{p}=.05$ showed that there was no difference in JOS ratings in the $\mathrm{CF}$ alternatives condition $(\mathrm{M}=$ $4.44, \mathrm{SD}=1.88)$ and in the Control condition $(\mathrm{M}=4.59, \mathrm{SD}=$ 1.78); thus, our manipulation did not affect reasoners' expectations of how well they would perform. In contrast, as predicted, mean Confidence was significantly higher in the CF alternatives condition $(\mathrm{M}=5.49, \mathrm{SD}=1.53)$ than in the Control condition $(\mathrm{M}=4.90, \mathrm{SD}=1.67)$. Similarly, analyses showed that mean Confidence did not differ significantly from the JOS rating in the Control condition, while significantly increasing in the $\mathrm{CF}$ alternatives condition.

Consistent with previous studies, prompting reasoners to generate potential alternative antecedents to an empirically false conditional relation improves abstract reasoning (Markovits, \& Lortie Forgues, 2011). As predicted, when participants are prompted to reason better on the abstract problems, they are aware of this improvement, which is reflected in higher Confidence ratings. Critically, the CF alternatives task does not directly tap into reasoners' explicit logical knowledge, so that the increase in Confidence cannot be attributed to learning of logical principles.

These data are certainly consistent with our hypothesis. There are, however, alternative explanations that must also be examined. The first is the possibility that the increase in mean Confidence ratings might be due to greater precision of the metacognitive evaluation process, that is, it might have increased the calibration between accuracy and confidence. To test this, we computed correlations between the total number of logically correct responses and mean Confidence ratings; these were as high in the Control condition, $\mathrm{r}(56)=.43, \mathrm{p}<$ .001 as in the CF alternatives condition, $r(59)=.47, \mathrm{p}<.001$. A second possible explanation is that the manipulation affected primarily people's confidence, which then produced an increase in logical responding. There are several reasons that suggest that this explanation is unlikely. First, if a generalized increase in confidence resulted from the manipulation, then this should have been observable in the initial JOS ratings. However, the data clearly showed that JOS ratings did not 
differ between the two conditions. Second, if there were a causal relationship between confidence and normative responding, this would also be apparent when examining reasoning with concrete content. In contrast, the results of Study 1 showed that higher ratings of confidence are not directly related to levels of normative responding. Thus although these results must be interpreted with caution, in combination with the results of the initial study, they provide strong evidence for the existence of a metacognitive representation related to logical validity.

We also examined more specific patterns of correlations between Confidence ratings and logical reasoning for each of the individual logical forms, combined across all participants. The correlations were significant and positive for three out of the four inferences (MP: $r(126)=.47, p<.001$; AC: $r(126)=$ $.36, p<.001$; MT: $r(126)=.22, p<.02)$ and marginally significant for the DA form, $r(126)=.17, p<.06$. In addition, the correlation between initial JOS rating and logical reasoning was globally positive, $\mathrm{r}(117)=.24, \mathrm{p}<.01$.

Finally, we examined correlations for individual inferences. These showed significant correlations for the two MP inferences $(r=.37, p<.001 ; r=.42, p<.001)$, and for the two AC inferences $(r=.32, p<.001 ; r=.24, p<.001)$. In addition, correlations for the MT inferences were positive, but only marginally so for the initial inference $(r=.14, p<.13$; $r=.24, p<$ .001 ), while correlations for the DA inferences were positive but not statistically significant ( $r=.08, p=$ n.s.; $r=.14, p=$ n.s. $)$.

\section{General discussion}

Surprisingly, despite an enormous body of work on the bases of confidence in other psychological domains, the analogous study of meta-reasoning processes is in its infancy (Ackerman \& Thompson, 2014; Thompson, 2009). Extant evidence shows that confidence in an inference or conclusion is sensitive to the fluency with which it is produced (Ackerman \& Zalmanov, 2012; Thompson et al., 2011, 2013a, b), selfperceived reasoning ability (Prowse Turner \& Thompson, 2009), and how much time is allotted to solve the problem (Thompson et al., 2011). In the current paper, we provided evidence for two new bases of confidence. Specifically, we have shown that the familiarity of the materials increases confidence in responses, even when it decreased the accuracy of those responses. We have also provided evidence for a more abstract standard of evaluation, by showing that people are able to evaluate the accuracy of their logical deductions with abstract materials; moreover, they are able to do so both prospectively (as shown by the correlation between JOS and confidence) and retrospectively (as shown by the correlation between confidence and accuracy). In contrast, however, when given concrete material to reason with, it appears that a different meta-representation is evoked, and that peoples' confidence judgments are mediated by the degree to which people's knowledge about the premises suggest normative responding. Thus, we have evidence for both knowledgeand validity-based meta-representations.

One might reasonably ask how people would be able to reason logically without a meta-representation of concepts such as validity. However, as we argued in the introduction, metacognitive theories (e.g., Nelson \& Narens 1990) generally distinguish between two types of processes: those that carry out basic cognitive functions such as memory and reasoning, and those that monitor those functions. These functions may operate quite differently. That is, one might produce a conditional inference by building a mental model of the conditional and generating a conclusion with a concomitant search for semantic counterexamples (Johnson-Laird \& Byrne, 2002), by assessing the conditional probability of the conclusion given the premises (Oaksford \& Chater, 2003), or by a combination of these strategies (e.g. Markovits, Brunet, Thompson, \& Brisson, 2013). None of these theories presuppose a metacognitive understanding of validity or logical necessity. Indeed, under the probabilistic model, computing logically valid inferences is explicitly not the goal of the inferential processes, which instead assesses degrees of belief in the conclusions given the premises and other background information (Oaksford \& Chater, 2003). In situations where people are confronted with abstract materials, which do not allow an easy assessment of belief, they must attempt to modify this probabilistic strategy to fit the task demands. Thus, according to this model, it is perfectly possible for reasoners to make inferences that sometimes correspond to the norms of logical validity, but with inferential machinery designed for a different purpose. In fact, as we indicated in the introduction, the disconnect between metacognitive understanding and inferential performance is specifically postulated by developmental theories such as that proposed by Moshman (1998). Consequently, an important and novel contribution of the current work is to show that reasoners have a meta-representation of logical validity that can be used to inform them of the accuracy of their logical deductions, at least when reasoning about abstract materials.

Moreover, it should be noted that previous work on the relationship between confidence and accuracy in reasoning has come to a much more pessimistic conclusion. Using complex reasoning problems, such as three-term syllogisms, two studies with large numbers of participants showed that reasoners were unable to tell when they had erred, showing equal levels of confidence for right and wrong answers (Prowse Turner \& Thompson, 2009; Shynkaruk \& Thompson, 2006). Training improved the accuracy of reasoning, but had a minimal effect on calibration (Prowse Turner \& Thompson, 2009). 
As we have argued above, one explanation for these findings that is consistent with the present results is that confidence judgments are often inferential (Ackerman \& Thompson, 2014; Koriat, 2007; Dunlosky \& Tauber, 2014) and use available cues, such as fluency and familiarity, which may not be reliable indicators of accuracy. For example, confidence generally increases with the amount of time spent thinking about a problem, even when the additional time does not increase accuracy (Shynkaruk \& Thompson, 2006; Thompson et al., 2011). Cues may also be derived from the way an item was processed: answers that come easily to mind engender confidence, even though ease of retrieval is not necessarily diagnostic of accuracy (Ackerman \& Zalmanov, 2012). Indeed, the current studies provide additional evidence of the role of such cues in the generation of confidence judgments. Reasoners were more confident when reasoning about familiar than abstract statements, even when the familiar content was misleading. That is, reasoners were more confident in both the Few and Many alternatives than in the Abstract condition, although only the Many alternatives facilitated performance. Thus, the familiarity of the material appears to be another cue to confidence, one that is not related to validity.

Our findings are particularly important in light of the claim that ordinary reasoners have "logical intuitions." For example, De Neys has observed that many aspects of reasoners' judgments, such as ability to recall problem information and confidence in a response, are sensitive to the conflict between logic/probability information and belief (see De Neys, 2012 for review). As belief information is usually presumed to be available quickly via Type 1 processing (e.g. Evans \& CurtisHolmes, 2005; Johnson-Laird \& Bara, 1984), these findings suggest that information about logic/probability is available fast enough to conflict with beliefs. Two recent studies have found additional evidence to support this conclusion: Handley, Newstead, and Trippas (2011) asked participants to judge either the believability or the validity of conditional inferences and found two-way interference of logical status on belief and vice versa; Pennycook, Trippas, Handley, and Thompson (2013) obtained similar findings with a base rate task. Our data suggest that people not only have "logical intuitions," but also have some access to metacognitive knowledge of the accuracy of those intuitions. Thus, in some circumstances, reasoners are able to both make logically correct inferences and treflect accurately on their performance. However, our results also show that confidence judgments are strongly affected by cues such as familiarity, which are not directly related to logical validity.

It is not clear, however, the extent to which this metacognitive knowledge is explicit. Our data do not offer an answer to this question. Many metacognitive judgments are based almost entirely on implicit cues, so that the participant can tell that they are more or less confident in an answer, but cannot articulate the basis of that answer; others more clearly reflect a combination of explicit and implicit factors (Koriat, 2007). The fact that JOSs predict performance suggests that there is at least some explicit basis for our reasoners' metarepresentation of abstract logic, but further study is needed before that conclusion can be drawn.

\section{Conclusions}

In this paper, we have made four novel discoveries about meta-reasoning: First, we demonstrated that confidence in reasoning inferences is moderated by the familiarity of the material that is being reasoned about, even when that familiarity reduces logical performance. This finding parallels those from the meta-memory literature (Costermans et al., 1992; Koriat \& Levy-Sadot, 2001) and is further evidence that the monitoring of reasoning processes, similar to memory processes, is inferential and based on cues. Second, we provided the first empirical data on a new type of meta-reasoning judgment, called the Judgment of Solvability, which was correlated with reasoning accuracy for abstract, but not concrete materials. On this basis, we concluded that reasoners' initial evaluation of their ability to make logical inferences draws on a validity-based meta-representation. Third, we provided evidence that, in addition to inferential cues such as familiarity and fluency, reasoners have access to a more abstract standard of evaluation that allows them to evaluate the logical validity of their inferences, at least when reasoning with concrete material. Finally, when reasoning with familiar material, reasoners confidence judgments reflected the properties of the materials they were reasoning with, such that it was higher when their inferences were consistent with prior knowledge and lower when they were not.

Acknowledgments This research was supported by a Discovery grant from the Natural Sciences and Engineering Council of Canada to H.M.

\section{References}

Ackerman, R., \& Thompson, V. A. (2014). Meta-reasoning: What can we learn from metamemory. In A. Feeney \& V. A. Thompson (Eds.), Reasoning as memory (pp. 164-182). UK: Psychology Press.

Ackerman, R., \& Zalmanov, H. (2012). The persistence of the fluencyconfidence association in problem solving. Psychonomic Bulletin \& Review, 19(6), 1189-1192.

Costermans, J., Lories, G., \& Ansay, C. (1992). Confidence level and feeling of knowing in question answering: The weight of inferential processes. Journal of Experimental Psychology: Learning, Memory, and Cognition, 18(1), 142.

Cummins, D. D., Lubart, T., Alksnis, O., \& Rist, R. (1991). Conditional reasoning and causation. Memory \& Cognition, 19(3), 274-282.

De Neys, W. (2012). Bias and conflict: A case for logical intuitions. Perspectives on Psychological Science, 7(1), 28-38.

De Neys, W., Cromheeke, S., \& Osman, M. (2011). Biased but in doubt: Conflict and decision confidence. PloS One, 6(1), e15954. 
De Neys, W., \& Glumicic, T. (2008). Conflict monitoring in dual process theories of thinking. Cognition, 106(3), 1248-1299.

De Neys, W., Rossi, S., \& Houdé, O. (2013). Bats, balls, and substitution sensitivity: Cognitive misers are no happy fools. Psychonomic Bulletin \& Review, 20(2), 269-273.

De Neys, W., Vartanian, O., \& Goel, V. (2008). Smarter than we think: When our brains detect that we are biased. Psychological Science, 19(5), 483-489.

Dunlosky, J., \& Tauber, S. K. (2014). Understanding people's metacognitive judgments: An isomechanism framework and its implications for applied and theoretical research. In T. Perfect \& D. S. Lindsay (Eds.), Handbook of applied memory (pp. 444-464). Thousand Oaks, CA: Sage.

Epstein, S. (1994). Integration of the cognitive and the psychodynamic unconscious. American Psychologist, 49, 709-709.

Evans, J. S. B. T. (2007). Hypothetical thinking: Dual processes in reasoning and judgement. New York, NY US: Psychology Press.

Evans, J. S. B., \& Curtis-Holmes, J. (2005). Rapid responding increases belief bias: Evidence for the dual-process theory of reasoning. Thinking \& Reasoning, 11(4), 382-389.

Franssens, S., \& De Neys, W. (2009). Belief inhibition during thinking: Not always winning but at least taking part. Cognition, 113(1), 45-61.

Frederick, S. (2005). Cognitive reflection and decision making. Journal of Economic Perspectives, 19(4), 25-42.

Galinsky, A. D., \& Moskowitz, G. B. (2000). Counterfactual as behavioural primes: Priming the simulation heuristic and consideration of alternatives. Journal of Experimental Social Psychology, 36, 384- 409.

Handley, S. J., Newstead, S. E., \& Trippas, D. (2011). Logic, beliefs, and instruction: A test of the default interventionist account of belief bias. Journal of Experimental Psychology Learning Memory and Cognition, 37(1), 28-43. doi:10.1037/a0021098

Heit, E., \& Rotello, C. M. (2010). Relations between inductive reasoning and deductive reasoning. Journal of Experimental Psychology: Learning, Memory, and Cognition, 36(3), 805.

Johnson-Laird, P. N., \& Bara, B. G. (1984). Syllogistic inference. Cognition, 16, 1-61.

Johnson-Laird, P. N., \& Byrne, R. M. J. (1991). Deduction. Hove \& London: Erlbaum.

Johnson-Laird, P. N., \& Byrne, R. M. J. (2002). Conditionals: A theory of meaning, pragmatics and inference. Psychological Review, 109, 646-678.

Karmiloff-Smith, A. (1995). Beyond modularity: A developmental perspective on cognitive science. Cambridge, MA: MIT Press.

Koriat, A. (2007). Metacognition and consciousness. In P. D. Zelazo, M. Moscovitch, \& E. Thompson (Eds.), The Cambridge handbook of consciousness (pp. 289-325). Cambridge, UK: Cambridge University Press.

Koriat, A., \& Levy-Sadot, R. (2001). The combined contributions of the cue-familiarity and accessibility heuristics to feelings of knowing. Journal of Experimental Psychology: Learning, Memory, and Cognition, 27(1), 34.

Markman, K. D., Lindberg, M. J., Kray, L. J., \& Galinsky, A. D. (2007). Implications of counterfactual structure for creative generation and analytic problem-solving. Personality and Social Psychology Bulletin, 33(3), 312-324.

Markovits, H. (2000). A mental model analysis of young children's conditional reasoning with meaningful premises. Thinking \& Reasoning, 6(4), 335-347.

Markovits, H., Brunet, M.-L., Thompson, V., \& Brisson, J. (2013). Direct evidence for a dual process model of deductive inference. Journal of Experimental Psychology: Learning, Memory, and Cognition, 39(4), 1213-1222.

Markovits, H., \& Lortie Forgues, H. (2011). Conditional reasoning with false premises facilitates the transition between familiar and abstract reasoning. Child Development, 82(2), 646-660. doi:10.1111/j.14678624.2010.01526.x
Markovits, H., Lortie Forgues, H., \& Brunet, M.-L. (2010). Conditional reasoning, frequency of counterexamples, and the effect of response modality. Memory \& Cognition, 38(4), 485-492.

Markovits, H., \& Vachon, R. (1989). Reasoning with contrary-to-fact propositions. Journal of Experimental Child Psychology, 47, 398412.

Markovits, H., \& Vachon, R. (1990). Conditional reasoning, representation, and level of abstraction. Developmental Psychology, 26(6), 942-951.

Morsanyi, K., \& Handley, S. J. (2012). Logic feels so good-I like it! Evidence for intuitive detection of logicality in syllogistic reasoning. Journal of Experimental Psychology-Learning Memory and Cognition, 38(3), 596.

Moshman, D. (1998). Cognitive development beyond childhood. In D. Kuhn \& R. Siegler (Eds.), Handbook of child psychology (5th ed., Vol. 2, pp. 947-978). New York: Wiley.

Moshman, D., \& Franks, B. (1986). Development of the concept of inferential validity. Child Development, 57, 153-165.

Nelson, T. O., \& Narens, L. (1990). Metamemory: A theoretical framework and new findings. In G. Bower (Ed.), The psychology of learning and motivation: Advances in research (Vol. 26, pp. 125173). San Diego, CA: Academic Press.

Oaksford, M., \& Chater, N. (2003). Conditional probability and the cognitive science of conditional reasoning. Mind \& Language, 18(4), 359-379.

Pennycook, G., Trippas, D., Handley, S. H., \& Thompson, V. A. (2013). Base rates: Both neglected and intuitive. Journal of Experimental Psychology: Learning, Memory, \& Cognition. doi:10.1037/ a0034887

Prowse Turner, J. A., \& Thompson, V. A. (2009). The role of training, alternative models, and logical necessity in determining confidence in syllogistic reasoning. Thinking \& Reasoning, 15(1), 69-100.

Rips, L. J. (2001). Two kinds of reasoning. Psychological Science, 12(2), $129-134$

Shynkaruk, J. M., \& Thompson, V. A. (2006). Confidence and accuracy in deductive reasoning. Memory \& Cognition, 34(3), 619-632.

Sloman, S. A. (1996). The empirical case for two systems of reasoning. Psychological Bulletin, 119(1), 3-22.

Stanovich, K. E., \& West, R. F. (2000). Individual differences in reasoning: Implications for the rationality debate? Behavioral and Brain Sciences, 23(5), 645-726.

Thompson, V. A. (1994). Interpretational factors in conditional reasoning. Memory \& Cognition, 22(6), 742-758.

Thompson, V. A. (2000). The task-specific nature of domain-general reasoning. Cognition, 76(3), 209-268.

Thompson, V. A. (2009). Dual process theories: A metacognitive perspective. In two minds: dual processes and beyond. Oxford University Press, Oxford.

Thompson, V. A., Evans, J. S. B. T., \& Campbell, J. I. D. (2013a). Matching bias on selection task: It's fast and it feels good. Thinking \& Reasoning, 19, 431-452.

Thompson, V. A., Prowse Turner, J. A., \& Pennycook, G. (2011). Intuition, reason, and metacognition. Cognitive Psychology, 63(3), $107-140$

Thompson, V. A., Turner, J. A. P., Pennycook, G., Ball, L. J., Brack, H., Ophir, Y., \& Ackerman, R. (2013b). The role of answer fluency and perceptual fluency as metacognitive cues for initiating analytic thinking. Cognition, 128, 237-251.

Topolinski, S. (2014). Intuition: Introducing affect into cognition. In A. Feeney \& V. A. Thompson (Eds.), Reasoning as memory (pp. 146163). UK: Psychology Press.

Winkielman, P., \& Cacioppo, J. T. (2001). Mind at ease puts a smile on the face: Psychophysiological evidence that processing facilitation leads to postive affect. Journal of Personality and Social Psychology, 81, 989-1000. 